\title{
Chantier ouvert au public
}

Marie-Pierre Julien, Julie Poirée, Céline Rosselin, Mélanie Roustan et Jean-Pierre Warnier

\section{OpenEdition}

\section{Journals}

Édition électronique

URL : https://journals.openedition.org/tc/1559

DOI : $10.4000 /$ tc. 1559

ISSN : 1952-420X

Éditeur

Éditions de l'EHESS

Édition imprimée

Date de publication : 1 avril 2003

ISSN : 0248-6016

\section{Référence électronique}

Marie-Pierre Julien, Julie Poirée, Céline Rosselin, Mélanie Roustan et Jean-Pierre Warnier, « Chantier ouvert au public », Techniques \& Culture [En ligne], 40 | 2003, mis en ligne le 07 juin 2006, consulté le 29 septembre 2022. URL : http://journals.openedition.org/tc/1559 ; DOI : https://doi.org/10.4000/tc.1559

Ce document a été généré automatiquement le 29 septembre 2022.

Tous droits réservés 


\title{
Chantier ouvert au public
}

\author{
Marie-Pierre Julien, Julie Poirée, Céline Rosselin, Mélanie Roustan et \\ Jean-Pierre Warnier
}

1 En voulant rouvrir ${ }^{1}$, il y a dix ans, le chantier de la « culture matérielle », les fondateurs du groupe "Matière à Penser" ne pressentaient pas dans quelles aventures ou mésaventures, de fouilles en consolidations, de consolidations en constructions, ils et elles s'embarquaient. Le chantier est là, ouvert au public (munissez-vous d'un casque : une brique foucaldienne, détachée d'un édifice instable, est susceptible de vous tomber sur la tête). Nous devons à la générosité et à la probité de Jean-Luc Jamard (2002) une visite guidée (état du chantier en 1999; en 2002, le paysage a déjà pas mal changé). Ses commentaires critiques sont justifiés : nos fouilles dans les strates de la pensée savante mettent à nu des matériaux éclectiques. Nos constructions font mur de tout concept comme on fait flèche de tout bois. Les échafaudages bricolés soutiennent des constructions incomplètes. Les gravats s'accumulent. Bientôt, il faudra ferrailler les échafaudages, paysager les déblais. Mais faudra-t-il cacher l'éclectisme des inspirations théoriques sous le doux gazon des expressions consensuelles, comme les architectes cachent leurs erreurs sous le lierre, les cuisiniers sous les sauces, les médecins sous le marbre...? En attendant, profitons du parcours commenté que propose Jean-Luc Jamard pour faire le bilan.

2 Nous en avons besoin pour notre propre gouverne. Le débat est là, grâce à Techniques \& culture et à son rédacteur en chef; qu'ils en soient chaleureusement remerciés.

Quels objets?

3 L'article et le schéma récapitulatif de J.-L. Jamard (2002, cf. figure, p. 232) jalonnent un espace sur lequel nous souhaitons revenir car ses limites sont précisément celles du débat possible: entre la technologie culturelle et une approche praxéologique de la culture matérielle, comment se situent respectivement les objets, les questions posées, les postures de recherche? En quoi se différencient-ils? Dans quelle mesure se recoupent-ils?

$4 \mathrm{Au}$ premier abord, nos thèmes de recherche ont peu en commun avec ceux de Techniques \& culture. Nos interrogations sont diverses, kaléidoscope éclectique de questions apparemment mineures : comment s'y prend-on pour habiter une pièce - 
quatre murs, une porte, et tout le confort dans le couloir? Qu'est-ce que cela fait à l'habitant, à l'habitation, et comment l'analyser (Rosselin 1999) ? Même question pour les «rippers " d'Emmaüs, sur les actions de trier, manipuler, casser, mettre en déchetterie ou recycler pour la vente vêtements, vaisselle, livres ou mobilier... Qu'estce que cela fait, non seulement à la vaisselle, mais aussi et surtout au compagnon qui la trie ou la casse (Hoarau 1999) ? Ou encore, comment les artisans chinois installés à Paris se construisent-ils comme laqueurs (Julien 2002)? Comment les pratiquants de jeux vidéo ou les consommateurs de cannabis se construisent-ils - ou non- en " e-sportifs " ou en "fumeurs de joints " ${ }^{2}$ ? Comment, enfin, le chercheur lui-même, dans son implication corporelle et son rapport aux objets, se construit-il comme ethnographe (Poirée 2002) ${ }^{3}$ ?

5 D'aucuns pourront aussi nous reprocher d'avoir construit la charpente avant les fondations. C'est que nous n'avons pas de modèle explicatif ou méthodologique posé $a$ priori et que nous testerions systématiquement. Dans la rencontre avec nos terrains respectifs, nous adaptons la méthode ethnographique, au risque de voir crouler l'édifice sous des thématiques non soupçonnées. D'où cette variété dans les échelles, d'où cette diversité dans les thèmes développés, d'où cette richesse aussi dans la mise en commun de nos objets.

6 Notre domaine d'élection n'est pas celui du travail dans le cadre de l'activité économique, de l'action sur la matière dans le laboratoire, l'atelier ou l'usine, mais celui de faits hétéroclites qu'on met en tas, sur lequel on plante, comme l'écrivait Marcel Mauss, un «jalon d'ignorance » portant la mention « divers». (Devinette : quel point commun y a-t-il entre un compagnon d'Emmaüs, une étudiante en chambre de bonne, un laqueur chinois à Paris, un joueur de jeux vidéo, un consommateur de cannabis et un ethnographe ?) C'est là qu'il y a des choses à trouver, aux frontières entre cognitivisme, ethnologie, praxéologie, psychologie, là où « les professeurs se bouffent entre eux ».

7 Le seul rapport entre ces activités, c'est qu'elles impliquent quatre éléments : une ou des personnes, des actions (ça bouge), des objets (les personnes agissent dans un rapport praxique aux choses) et du collectif, qui confèrent récurrence et prévisibilité aux répertoires de conduites et à l'usage des choses, autrement dit une inscription des actions dans le social ou une institutionnalisation. Il s'agit en général d'activités triviales, jouissant d'une faible légitimité scientifique ${ }^{4}$.

8 Nous pensons, à tort ou à raison, que l'objet qui intéresse la technologie culturelle partage ces quatre caractéristiques, mais que l'ajout d'une cinquième, qui est celle du travail technique comme action efficace sur la matière, en y concentrant son champ d'investigation, le réduit (cf. Julien \& Rosselin, ce volume). Pourtant, il existe une large zone de recoupement de nos objets de recherche, et c'est cette zone qui délimite le débat. Il nous semble que sur ces limites, il y a consensus, chacun ayant sa manière de l'exprimer.

Une subjectivation sans sujet?

9 Le sujet fait débat y compris au sein du MàP. C'est une des difficultés majeures soulignées par J.-L.Jamard (2002: 236). Et c'est autour de la cinquième dimension signalée à l'instant que la difficulté se noue. En technologie culturelle, ce qui est novateur, fécond, et qui a opéré un apport fondamental, c'est le problème de la configuration sociale et culturelle des techniques, ou, vice versa, de la configuration technique de la culture et de la société. Champ énorme. La question réflexive est nécessairement repoussée au second plan, en particulier du fait de la difficulté 
d'accorder la singularité avec le social (pace la querelle des universaux -Jamard 2002 : 218). Un « sujet social » serait-il un oxymore? Non, dirait Michel Foucault (1994: 227), dans la mesure où un sujet est assujetti :

«Il y a deux sens au mot "sujet": sujet soumis à l'autre par le contrôle et la dépendance, et sujet attaché à sa propre identité par la conscience ou la connaissance de soi. Dans les deux cas, ce mot suggère une forme de pouvoir qui subjugue et assujettit. ».

Cette dimension du travail sur soi, et donc d'une intériorité ou d'une subjectivité, est au centre de nos préoccupations.

11 Un « acteur social », bien évidemment, n'est pas un oxymore. Et cependant, Jean-Luc Jamard (2002: 237, note 28) nous rappelle, références à l'appui, que la technologie culturelle n'a pas évacué le travail sur soi que doit accomplir le travailleur. Sans doute, pour mettre les points sur les « $\mathrm{i} »$, avons-nous minimisé cet apport.

12 Foucault laisse dans l'ombre des procédés de subjectivation qui donnent une grande place au corps et notamment ceux qui rendraient opératoire la notion de subjectivation dans d'autres cultures, bien qu'il ait écrit :

«Je crois que les techniques de soi peuvent être trouvées dans toutes les cultures sous différentes formes. De même qu'il est nécessaire d'étudier et de comparer les différentes techniques de production des objets et la direction des hommes par les hommes à travers la forme d'un gouvernement, on doit aussi interroger les techniques de soi » (1994: 409).

13 Cette «technologie de soi » (Foucault $1994: 215)$ qui est selon nous mise en œuvre à travers les techniques d'objets, et donc à travers les techniques du corps (Julien \& Warnier 1999), nous a permis d'engager un dialogue fécond avec les praxéologues (Parlebas 1999; During 1984).

14 C'est sur ce point que nous souhaitons avancer dans le débat, car de trois choses l'une : quand il y a des humains, de l'action, de la matière et des répertoires institutionnalisés, ce qui se passe affecte soit le ou les sujets, soit les objets, soit les deux. Ce «les deux" en rapport dialectique ( $c f$. Hegel, Marx et leur postérité) retient toute notre attention, et nous y cherchons les principes d'un monisme que nous tentons de cerner autour des notions de sujet et de subjectivation. Nous avons sur ce point plusieurs positions. En effet, certains d'entre nous pensent qu'il est heuristiquement payant de récupérer la notion de sujet et de lui offrir un ravalement de façade, jusqu'à en faire, pourquoi pas, ce sujet vide et «sans qualité, que la science requiert »-le sujet lacanien. À l'opposé, d'autres membres du groupe, à la suite de Jean-François Bayart (s/presse) $)^{5}$, préfèrent penser une subjectivation sans sujet (en s'alignant sur le deuxième Foucault) -en d'autres termes un processus jamais achevé. Notre anthropologie du sujet -laquelle laisse Jamard perplexe (2002: 218) - est donc plutôt une anthropologie des subjectivations qui nous permet d'analyser par le singulier la construction des individus dans leur rapport dynamique à la matière et aux autres ${ }^{6}$.

15 À l'arrière-plan, deux débats se dessinent. Le premier intéresse la dimension politique de la subjectivation. Il n'est pas explicitement soulevé par notre « discutant »; laissonsle donc de côté mais il faudra y venir un jour. Le second est celui du monisme. Il occupe une place de choix dans les préoccupations de la technologie culturelle et de Jamard. Oui, bien sûr, encore un petit effort pour être vraiment latourien. Voilà un idéal à atteindre (qui ne semble pas vraiment être celui du dernier cité), mais comment ? Bruno Latour s'y emploie en logeant les humains et les non-humains à la même 
enseigne du collectif qu'ils forment ensemble, et avec des statuts aussi proches que possible l'un de l'autre. Nous explorons une voie différente, qui est celle de la subjectivation, avec ou sans sujet. Disons-le en termes de "sujet »: il n'existe aucun sujet qui ne se meuve, ne perçoive et n'éprouve des affects, ni aucune action qui ne s'étaye sur une culture matérielle faite de main d'homme, de sorte que les objets sont toujours des objets mis ou démis en sujets, et les sujets, des sujets mis ou démis en objets dans l'action. En termes de subjectivation, pas de subjectivation sans action, perception, affects, objets et institutionnalisation.

Quels sont les bénéfices et les pertes de chacune des deux postures (latourienne et mapiste) ? Nous ne nous prononcerons pas sur la première position, mais, quant à nous, il nous semble qu'à partir de la nôtre, nous pouvons mobiliser toutes les recherches en science de l'action motrice, en neurosciences, sur la perception, la mise en jeu des sens, l'incorporation, la prise, etc., qui nous permettront, espérons-le, de comprendre et d'analyser finement, dans le détail, ce qui se passe dans le collectif « sujet-et-ses-objetsen-action ». L'enjeu est d'analyser une subjectivation socialisée ou encore institutionnalisée, avec toutes les variantes individuelles que cela comporte. Pour l'instant, nous ne voyons pas dans quel sens trancher le débat, et quelle est la voie qui, à terme, débouchera. Nous serions encore tentés -en inversant la formule ironique de Jamard (2002: 240) - de dire sotto voce: «Allons, amis latouriens, encore un petit effort pour être vraiment mapistes! ».

Et l'éclectisme?

Hélas, oui, nous le confessons, nous sommes impurs et coupables de mélange... À l'éclectisme des terrains d'enquête et de leur approche, nous ajoutons l'éclectisme des sources d'inspiration théorique. De Marcel Mauss 1950) à Pierre Parlebas (1999), de Alain Berthoz (1997) à Pierre Bourdieu (1980), de Henry Head et Georges Holmes (1911) à Michel Foucault (1994), nous sommes disposés à nous approprier les travaux de toutes celles et tous ceux qui peuvent nous aider à réfléchir de façon anthropologique sur les choses matérielles, les conduites motrices, la perception, les affects, la subjectivation. Cela fait beaucoup de monde, beaucoup de notions hétéroclites, beaucoup de jargon. D'ailleurs, même si « la sociologie est un sport de combat » (film sur Bourdieu sorti en 2001), le vocabulaire de la praxéologie des activités ludiques et sportives, justifié dans le cas de ces activités, doit sans doute être expliqué, sinon employé avec parcimonie en dehors de sa terre d'élection. Il en est de même pour celui de la psychanalyse.

Sous ces multiples éclairages, l'objet semble aussi diffus qu'éclaté. Pour l'instant, nous en sommes au stade des traductions et équivalences (que l'on songe aux glissements de sens et aux subtiles nuances entre "ethos", "habitus", "habitudes", " hexis", « conduite », « comportement », «style de vie»). Mais notre démarche ne part pas de rien. Elle s'inscrit dans la rencontre de multiples traditions anthropologiques qui, à la suite de Marcel Mauss et d'André Leroi-Gourhan (1971), prennent en compte l'humain en action.

L'action: nous sommes bien devant la pierre de touche du débat avec Techniques \& culture; c'est là, dans une commune référence à André Leroi-Gourhan, que se situe pour nous l'apport le plus précieux du dialogue. Dans cette optique, et au vu des remarques de notre discutant, la deuxième partie de Construire la culture matérielle (Warnier 1999), qui avait pour ambition d'établir le dialogue avec l'anthropologie de la consommation et les études sur l'imaginaire du matériel, perd tout relief. Elle nous ramène néanmoins à la question du sens. 
20 Lors de la constitution du MàP, au cœur même de sa fondation pourrait-on dire, nous avions choisi d'isoler la dimension symbolique du rapport aux choses matérielles, de la nier pour un temps, délibérément. Les approches par le symbolique, nous semblait-il, étaient déjà fort nombreuses: les travaux d'anthropologie phénoménologique, dont nous nous démarquons, ont ainsi dérivé vers l'exclusivité des significations et des représentations attachées au corps, laissant un vide là où des concepts seraient nécessaires à une ethnographie de la motricité dans le monde matériel; les travaux de David Le Breton (2001), prenant en compte le corps uniquement comme "objet» symbolique, ne peuvent pas nous aider à considérer la matérialité du corps et de l'action; de son côté, la sémiologie définit le corps comme instrument d'expression, un corps davantage support d'un système de signes au service de la vie sociale qu'agent sensori-moteur de construction de la réalité sociale.

21 Notre choix de découpage de la réalité nous permet de nous focaliser sur les pratiques, les objets dans leur matérialité, l'action incarnée ${ }^{7}$. C'est un moyen de choisir clairement un axe de recherche, une autre voie. Celle-ci nous a conduit à envisager les techniques du corps comme des techniques d'objets, puis des techniques de soi.

22 De la pratique, de l'action, peut alors émerger du sens, y compris du sens matériel ( $c f$. Warnier 1999). La suite, espérons-nous, au prochain colloque ou aux prochains volumes thématiques.

23 NdlR : Le débat est engagé; il faut s'en réjouir, car discussions et controverses de bon aloi se font rares dans nos prytanées. Souhaitons donc que d'autres échanges s'ensuivent pour notre profit à tous.

24 Jean-Luc Jamard

\section{BIBLIOGRAPHIE}

Bayart, Jean-François

S/presse. « Total subjectivation », in J.-F. Bayart et J.-P. Warnier (eds), Culture matérielle et subjectivation politique. Paris : Céri-Karthala.

Berthoz, Alain

1997. Le Sens du mouvement. Paris : Odile Jacob.

Bourdieu, Pierre

1980. Le Sens pratique. Paris : Les Éditions de Minuit.

Candau, Joel

2000. Mémoire et expériences olfactives. Anthropologie d'un savoir-faire sensoriel. Paris : Presses Universitaires de France (« Sociologie d'Aujourd'hui »).

During, Bertrand

1984. Des jeux aux sports. Paris : Vigot.

Foucault, Michel

1994. Dits et écrits, 1954 - 1988, Tome IV, 1980 - 1988. Paris : Gallimard (« N.R.F. »). 
Head, Henry et Holmes, Georges

1911. « Sensory Disturbances from Cerebral Lesions », Brain 1911-1912 : 34-102.

Hoarau, François

1999. « Trier, transporter à Emmaüs. Ethnographie, sens et sciences de l'action », pp. 97-106, in

M.-P. Julien et J.-P. Warnier (eds), Approche de la culture matérielle. Corps à corps avec l'objet. Paris :

L'Harmattan (« Connaissance des hommes »).

Jamard, Jean-Luc

2002. «Au cœur du sujet : le corps en objets », Techniques \& culture 39 : 211-247.

Julien, Marie-Pierre

2002. Les meubles laqués chinois made in France. Thèse de doctorat de 3ème cycle. Paris V/

Sorbonne (multicopié).

Julien, Marie-Pierre \& Jean-Pierre Warnier (eds)

1999. Approches de la culture matérielle : corps à corps avec l'objet. Paris : L'Harmattan

(«Connaissance des hommes»).

Latour, Bruno

1991. Nous n'avons jamais été modernes. Essai d'anthropologie symétrique. Paris : La Découverte.

Le Breton, David

1999. L’Adieu au corps. Paris : A.-M. Métaillé (« Traversées »).

Leroi-Gourhan, André

1971. L'Homme et la matière. Paris : Albin Michel (1ère édition 1943).

Mauss, Marcel

1950. «Les techniques du corps », pp. 365-386, in Marcel Mauss, Sociologie et Anthropologie. Paris :

Presses Universitaires de France (« Quadrige »).

Parlebas, Pierre

1999. Jeux, sports et sociétés. Lexique de praxéologie motrice. Paris : INSEP-Publications

(« Recherche»).

Poirée, Julie

2002. Techniques du corps et techniques de soi. Essai d'analyse praxéologique d'un rituel thérapeutique vodun à travers l'expérience ethno-vidéographique dans un couvent de Cotonou (Bénin). DEA Paris V/Sorbonne (multicopié).

Rosselin, Céline

1999. « Si tu vas un peu brusquement, tu te cognes contre l'armoire », pp. 107-118, in Julien M.-P.

et J.-P. Warnier (eds), Approche de la culture matérielle. Corps à corps avec l'objet ». Paris :

L'Harmattan («Connaissance des hommes »).

Warnier, Jean-Pierre

1999. Construire la culture matérielle. L'homme qui pensait avec ses doigts. Paris : Presses

Universitaires de France (« Sciences sociales et sociétés»).

\section{NOTES}

1. Nous écrivons « rouvrir ", car ce chantier fut déjà bel et bien ouvert, sous cette enseigne, à la fin du XIXe siècle. Il n'a jamais été clos de l'autre côté de la Manche, où les Notes and Queries in Anthropology comportent un dernier chapitre intitulé « Material 
Culture » et où certaines universités (ainsi University College, London) offrent des cursus et des diplômes sous cette étiquette.

2. Mélanie Roustan, thèse de doctorat en cours.

3. Ces questions se posent aussi à l'extérieur du MàP, notamment chez Romain Bertrand qui se demande comment analyser l'art du vêtement et les pratiques corporelles ascétiques des Priyayi indonésiens (2000, thèse de doctorat IEPP).

4. P. Parlebas ( $1999: 5$, et communication personnelle, Jean-Pierre Warnier) témoigne des difficultés qu'il a éprouvées à la fin des années 1970, et qu'il éprouve encore à un moindre degré au début des années 2000 , à faire admettre la motricité et les activités ludiques et sportives comme des objets scientifiques légitimes en sociologie. Il a fallu qu'il réfléchisse non seulement à l'objet, mais, dirait-on en « info-com », à des stratégies de communication afin de le faire accepter comme un objet sociologique digne d'intérêt. Nous sommes reconnaissants à J.-L. Jamard de considérer que la praxéologie de la culture matérielle est digne d'intérêt, malgré la pauvreté de nos stratégies de communication.

5. À paraître 2002, sous le titre « Total subjectivation ».

6. Le recueil des données ethnographiques ne s'appuie-t-il pas sur des informations issues d'êtres singuliers? Sur les rapports entre êtres singuliers et expériences partagées? (Cf. Candau 2000).

7. Nous souhaitons que le lecteur considère l'association de ces termes comme un pléonasme.

\title{
AUTEURS
}

\section{MARIE-PIERRE JULIEN}

Groupe MàP, UMR «LMS ». Université Paris V - René Descartes

\section{JULIE POIRÉE}

Groupe MàP, UMR « LMS ». Université Paris V - René Descartes

CÉLINE ROSSELIN

Groupe MàP, UMR «LMS ». Université Paris V - René Descartes

\author{
MÉLANIE ROUSTAN
}

Groupe MàP, UMR «LMS ». Université Paris V - René Descartes

JEAN-PIERRE WARNIER

Groupe MàP, UMR «LMS ». Université Paris V - René Descartes 